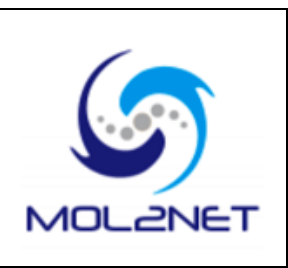

\title{
Modelling and simulation of SAW delay line sensors with COMSOL Multiphysics
}

\author{
Bilel Achour ${ }^{1}{ }^{*}$, Nadia Aloui ${ }^{1}$, Najla Fourati ${ }^{2}$, Chouki Zerrouki ${ }^{2}$, Nourdin Yaakoubi ${ }^{1}$ \\ 1 LAUM, UMR CNRS 6613, Le Mans Université, Avenue Olivier Messiaen, Le Mans,72085, \\ France; E-Mails: Nadia.Aloui@ univ-lemans.fr; Nourdin.Yaakoubi@ univ-lemans.fr; \\ 2 SATIE, UMR CNRS 8029, Cnam, 292 Rue Saint-Martin, Paris, 75003, France; E-Mails: \\ najla.fouratiennouri@lecnam.net; chouki.zerrouki@lecnam.net; \\ * Author to whom correspondence should be addressed; E-Mail: bilel.achour.etu@univ-lemans.fr; \\ Tel.: +33(0)7 69470265
}

Received: / Accepted: / Published:

\begin{abstract}
This study concerns 2D and 3D Finite Element Method (FEM) simulation of surface acoustic wave (SAW) sensors using COMSOL Multiphysics software. SAW device has been designed on piezoelectric substrate; $36^{\circ}$ rot lithium tantalate $\left(\mathrm{LiTaO}_{3}\right)$. Simulations were made on well-known structure to ensure the concordance between 2D and 3D models, and to define a 2D one that can account for and predict the electrical behaviour of SAW transducers for the future optimizations. The results show good agreement between numerical simulation and experimental S21 spectra. Accordingly, we can use the 2D built model for simulations intended to optimize the structure of devices, mainly for increasing their sensitivity.
\end{abstract}

Keywords: Finite Element Method (FEM); COMSOL Multiphysics; Surface acoustic wave (SAW); 36 lithium tantalate $\left(\mathrm{LiTaO}_{3}\right)$

\section{Introduction}

Modeling is of primary interest in many areas. It allows suitable optimization of components or structures before the realization steps, offering thus a substantial gain in both time and money. The field of sensors, and particularly Surface Acoustic Wave ones, is mostly concerned. Indeed, SAW devices can be found in wide domains, including physical sensors (temperature, pressure, torque, strain...) chem- and bio-sensors (insecticides, pollutants, proteins, biomarkers...), electronics, telecommunication, signal processing (filtering, modulation, RFID...).

The main quality of SAW sensors is their versatility, as they can function in either liquid or gaseous environments, for the detection of various analytes, such as DNA or analogues [1-2], antibodies/antigens [3], gases [4], pesticides [5], heavy metals [6], etc. Moreover, detection of chemical or biological entities can be done in real time and without labeling. Compared to other chemical and biological sensors, the SAW ones are highly sensitive and can achieve very low limits of detection [7-8], which explains the fact that they are still the subject of constant development. Therefore, simulation becomes a powerful tool to efficiently accompany these developments, to design optimized structure for applications were highly sensitive devices are needed. In this paper, we present a comparison between the numerical simulation and experimental results concerning a delay-line structure. This will permit us to define a model that can account for and predict the electrical behavior of any SAW transducers for the future optimizations. 
Mol2Net-04, 2018, BIOCHEMPHYS-01 (pages 1- x, type of paper, doi: xxx-xxxx http://sciforum.net/conference/mol2net-4

\section{Results and Discussion}

3D Finite Element Method (FEM) simulation was considered to simulate the physical and electrical behavior of SAW delay-line, using COMSOL software. The frequency response is obtained by considering the Delta-Function model whose equations are implemented in COMSOL. The key required parameter is the SAW velocity, that defined the delay-line operating frequency.

The simulations are run from $80 \mathrm{MHz}$ to $200 \mathrm{MHz}$ with a frequency step of $1 \mathrm{MHz}$. Calculated and experimental frequency responses (S21 spectra) are plotted in Fig. 1a and Fig.1b respectively. Numerical simulation results show that the delay-line operates around $104 \mathrm{MHz}$ as expected, but the maximum gain value $(-55 \mathrm{~dB})$ is lower than the experimental one $(-18 \mathrm{~dB})$. This is due to the fact that only 2 pairs of double fingers for both input and output interdigital transducers (IDTs) were used with COMSOL Multiphysics, while the real SAW device is constituted of 30

pairs fingers for each IDT. The chief benefit of simulations being the time saving, it was not possible to model a 3D response with 30 pairs of fingers, the calculation being very long, without guarantee of calculus convergence. Therefore, we redone $2 \mathrm{D}$ and $3 \mathrm{D}$ calculations on 2 and 3 pairs of fingers to make sure that the obtained results were comparable. Once ensured of results concordance, we simulated a 2D structure with 30 pairs of fingers. The results of the calculations, which lasted only few hours, are shown in Fig. 1.c. S21 spectra presents a comparable shape as experimental one: the characteristic frequency of SAW is clearly identified (with an insertion loss of $-21 \mathrm{~dB}$ ). Moreover, calculation equally account for fast volume waves around $140 \mathrm{MHz}$. Based on this calculation approach, several optimized structures will be tested before to manufacture new SAW sensors, sensitive enough to perform chemical and biological applications.
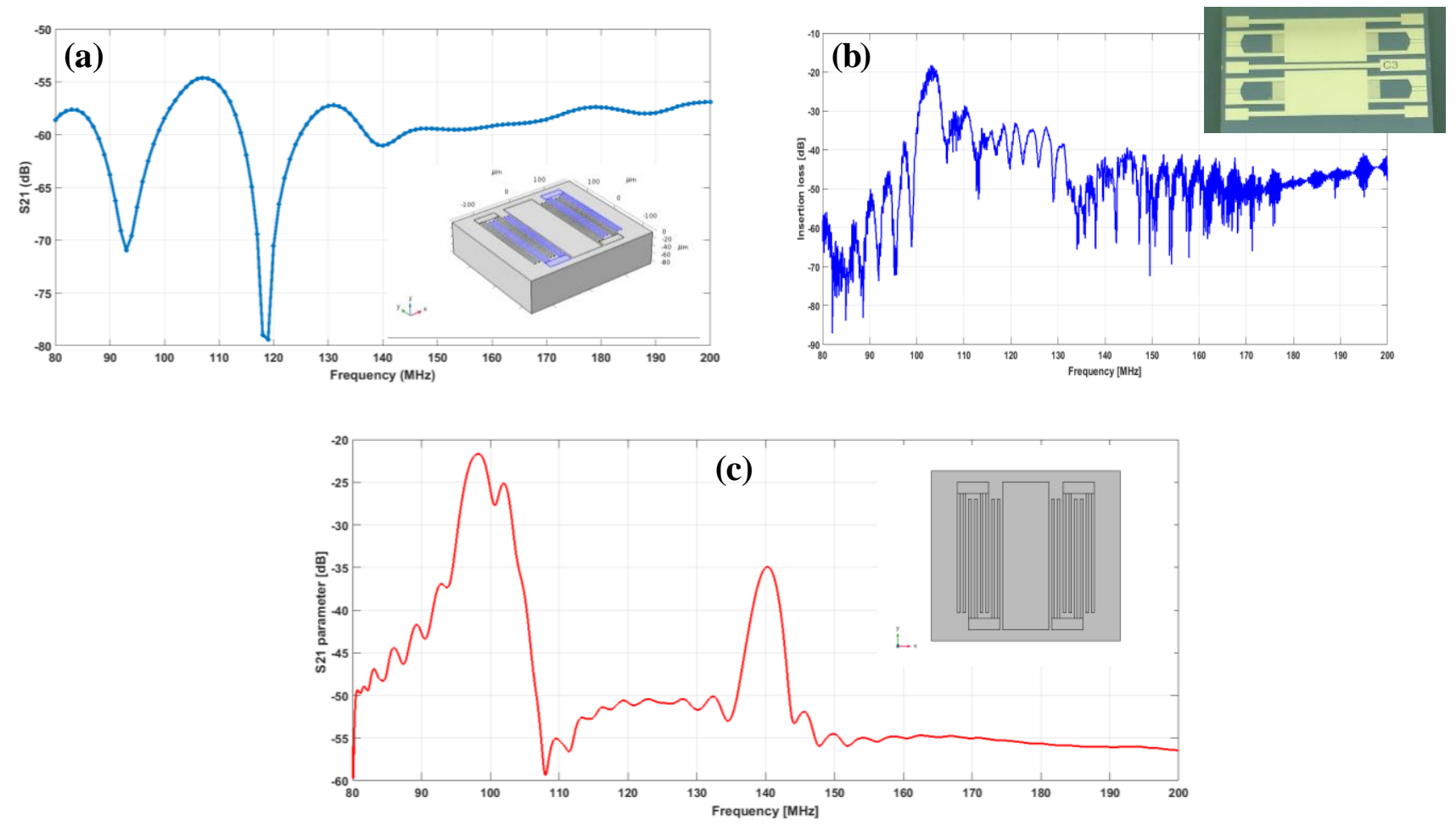

Figure 1. (a) Calculated S21 spectrum (3D model, 2 pairs of fingers for input and output IDTs); (b) experimental S21 spectrum; (c) Calculated S21 spectrum (2D model, 30 pairs of fingers for input and output IDTs). 
Mol2Net-04, 2018, BIOCHEMPHYS-01 (pages 1- x, type of paper, doi: xxx-xxxx http://sciforum.net/conference/mol2net-4

\section{Materials and Methods}

A SAW delay line was designed on $36^{\circ} \mathrm{LiTaO}_{3}$ piezoelectric substrate. Two IDTs were patterned in $\mathrm{Cr} / \mathrm{Au}(20 \mathrm{~nm} / 80 \mathrm{~nm})$ layers with the same periodicity of $\lambda=40 \mu \mathrm{m}$ (i.e. corresponding frequency of $104 \mathrm{MHz}$ ). The width of each finger is equal to $5 \mu \mathrm{m}$ [9]. A metallized $\mathrm{Cr} / \mathrm{Au}(20$ $\mathrm{nm} / 80 \mathrm{~nm}$ ) sensing area of $80 \mu \mathrm{m}$ length separates the input and output IDTs (Fig.2).

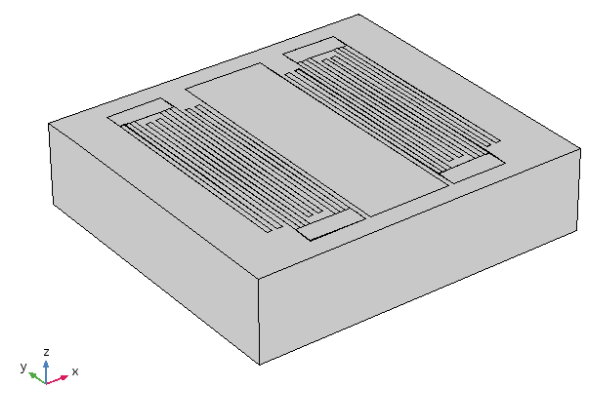

Figure 2. SAW sensor geometry.
Extra fine triangle elements and a swept mesh with a distribution are used for meshing the geometry of our SAW delay Line. The meshed model is shown in Figure 3.

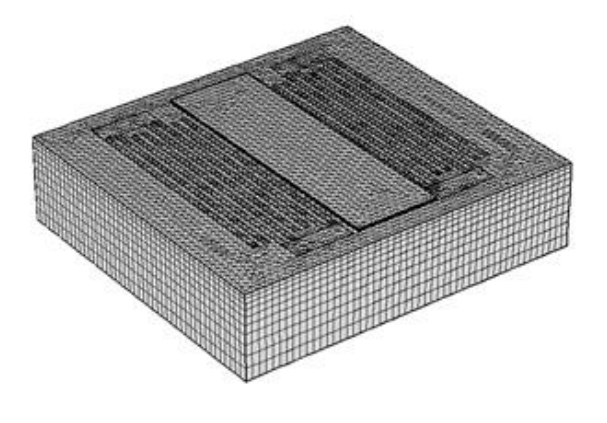

Figure 3. Mesh for simulation.

\section{Conclusions}

In this work, we have used COMSOL Multiphysics software to design and optimize the geometry of a SAW delay-line, and to determine its frequency response (S21 spectra). We have shown that 2D calculation can be used instead of 3D one without any information loss, leading to an important time saving. We observed a good correlation between 2D simulation and experimental results. This is of importance as it permits to envisage modelling of any structure before realization steps. This pad the way for new optimized designs of sensors, including adjunction of suitable materials between input and output IDTs, to confine the SAW energy close to the surface, enhancing thus their sensitivity.

\section{Acknowledgments}

N. Yaakoubi and B. Achour wish to thank "La région Pays de la Loire" for the financial support through the WISE / RCSF project.

\section{Conflicts of Interest}

The authors declare no conflict of interest.

\section{References}

1. Hur, Y.; Han, J.; Seon, J.; Pak, Y.E.; Roh, Y. Development of an SH-SAW sensor for the detection of DNA hybridization. Sens. Actuators Phys, 2005, vol. 120, no. 2, pp. 462-467, doi:10.1016/j.sna.2005.01.027.

2. Zerrouki, C.; Fourati, N.; Lucas, R.; Vergnaud, J.; Fougnion, J.M.; Zerrouki, R.; Pernelle, C. Biological investigation using shear horizontal surface acoustic wave sensor: small "Click generated" DNA hybridization detection, Biosens. Bioelectron, 2010, vol. 26, n 4, pp. 1759-1762, doi: 10.1016/j.bios.2010.08.036.

3. Rupp, S.; von Schickfus, M.; Hunklinger, S.; Eipel, H.; Priebe, A.; Enders, D.; Pucci, A. A shear horizontal surface acoustic wave sensor for the detection of antigen-antibody reactions for medical diagnosis. Sens. Actuators B Chem, 2008, vol. 134, no. 1, pp. 225-229, doi:10.1016/j.snb.2007.06.035.

4. Marcu, A.; Viespe, C. Surface Acoustic Wave Sensors for Hydrogen and Deuterium Detection. Sensors, 2017, vol. 17, no. 6, p. 1417, doi:10.3390/s17061417. 
5. Mazouz, Z.; Rahali, S.; Fourati, N.; Zerrouki, C.; Aloui, N.; Seydou, M.; Yaakoubi, N.; Chehimi, M.; Othmane, A.; Kalfat, R. Highly Selective Polypyrrole MIP-Based Gravimetric and Electrochemical Sensors for Picomolar Detection of Glyphosate. Sensors, 2017, vol. 17, no. 11, p. 2586, doi:10.3390/s17112586.

6. Ramshani, Z.; Reddy, A.S.G.; Narakathu, B.B.; Wabeke, J.T.; Obare, S.O.; Atashbar, M.Z. SHSAW sensor based microfluidic system for the detection of heavy metal compounds in liquid environments, Sens. Actuators B Chem, 2015, vol. 217, pp. 72-77, doi:10.1016/j.snb.2014.12.026.

7. Richardson, M.; Sankaranarayanan, S.K.R.S.; Bhethanabotla, V.R. Low Insertion Loss and Highly Sensitive SH-SAW Sensors Based on $36^{\circ} \mathrm{YX} \mathrm{LiTaO}_{3}$ Through the Incorporation of Filled Microcavities, IEEE Sens. J, 2015,vol. 15, no. 2, pp. 787-796, doi:10.1109/JSEN.2014.2353794.

8. Li, S.; Wan, Y.; Su, Y.; Fan, C.; Bhethanabotla, V.R. Gold nanoparticle-based low limit of detection Love wave biosensor for carcinoembryonic antigens. Biosens. Bioelectron, 2017, vol. 95, pp. 4854, doi:10.1016/j.bios.2017.04.012.

9. N. Fourati, J.M. Fougnion, L. Rousseau, P. Lepeut, O. Français, P. Boutin, C. Vederine, J.J.Bonnet, B. Mercier and C. Pernelle, Surface Acoustic Love Waves Sensor For Chemical And Electrochemical Detection, Proceedings of ESDA 2006: 8th Biennial Conference on Engineering Systems Design and Analysis, Torino, Italy, in CD-rom, Juillet, 2006. 